\title{
Cardiovascular, plasma catecholamine, and corticosterone responses to alterations in the predictability of electric shock in the rat
}

\author{
JAMES E. LAWLER, RONALD H. COX, GREGORY F. BARKER, \\ JOHN W. HUBBARD, and BRIAN J. SANDERS \\ University of Tennessee, Knoxville, Tennessee
}

\begin{abstract}
Although rats show a clear behavioral preference for predictable versus unpredictable cutaneous electric shock, there have been few studies that have addressed the cardiovascular and sympathoadrenal correlates of alterations in the predictability of aversive events. The current experiments sought to measure systolic and diastolic blood pressure, heart rate, and plasma levels of norepinephrine (NE), epinephrine (E), and corticosterone (CORT) in three groups of rats in conditions that differed in the predictability of shock in the presence or absence of a tone. It was predicted, in line with all the major theories of preference for signaled shock, that rats receiving unpredictable shock would show the greatest elevation in cardiovascular and sympathoadrenal function. In the first experiment, contrary to expectation, rats receiving the most predictable electric shocks showed the greatest elevations in systolic blood pressure. In Experiment 2, these findings were extended to diastolic blood pressure as well. Heart rate, on the other hand, revealed no differences between complete predictability and no predictability groups. However, heart rate was significantly higher in the group receiving intermediate predictability, effects which could be due to baroreceptor function. Although plasma catecholamines revealed no group differences, CORT showed a trend for the intermediate group to be the highest. These data are discussed with reference to other physiological studies on, and to theoretical explanations of, the preference for signaled shock.
\end{abstract}

Given a choice, rats prefer signaled (predictable) over unsignaled (unpredictable) electric shock. This phenomenon has been repeatedly demonstrated using a variety of approaches (Abbott \& Badia, 1979; Badia, Culbertson, \& Lewis, 1971; Lockard, 1963) recently reviewed by Badia, Harsh, and Abbott (1979). Although there are some disagreements regarding methods both for the demonstration of preference (e.g., Biederman, Furedy, \& Beatty, 1981 ) and for shock delivery (Furedy \& Biederman, 1976; Weiss, 1977), the phenomenon is nevertheless observable across many different experimental situations. Recently, a preference for signaled shock has been found in a procedure designed to overcome these methodological problems (Biederman et al., 1981).

Several theoretical approaches have been developed to account for the preference for signaled shock. One ap-

This research was supported by grants from the NIH (HL-19680) and the American Heart Association, Tennessee Affiliate, both to J. E. Lawler, and by a Biomedical Sciences Support Grant (7088) to the University of Tennessee, Knoxville. R. H. Cox was supported by a postdoctoral fellowship, HL-06036, from the USPHS. J. W. Hubbard is now in the Department of Pharmacology, University of Texas Health Sciences Center, San Antonio, Texas. J. E. Lawler is Research Investigator, American Heart Association, Tennessee Affiliate. Requests for reprints should be sent to him at: Department of Psychology, University of Tennessee, Knoxville, TN 37996. proach is the preparatory response hypothesis (Perkins, 1968). Signals are preferred because they allow the animal either to engage in behaviors, such as postural adjustments (Furedy \& Biederman, 1976), or to produce internal adjustments, such as endorphin release (Fanselow, 1979), which reduce the aversiveness of the situation.

The safety signal hypothesis emphasizes that the absence of a signal that reliably predicts shock can serve as a safety signal (Seligman, 1968). Rats prefer signaled shock because a reliable safety period reduces the total aversiveness of the situation (Seligman, Maier, \& Solomon, 1971).

A third hypothesis is that offered by Rescorla and Wagner (1972). This approach emphasizes classical conditioning of fear cues. A signal associated with shock competes with other environmental cues to reduce the conditioning of fear to the context.

Although none of these approaches makes a specific prediction regarding the effects of predictable or unpredictable shock on physiological functioning, there have been a number of studies that have examined whether alterations in predictability affect physiological function. Although the cardiovascular and sympathoadrenal systems are extremely sensitive to psychological manipulations (e.g., Lawler, Barker, Hubbard, \& Schaub, 1981; Bassett, Cairncross, \& King, 1973), no studies have examined either blood pressure or catecholamine responses in 
groups of animals that differ in the predictability of shock. However, several studies have measured other aspects of cardiovascular function. For example, part of the Caul, Buchanan, and Hays (1972) study focused on heart rate. The hypothesis was that uncertainty in the rat leads to bradycardia. Although five groups of animals were examined in that study, only two were directly relevant to the predictability issue-a completely predictable group (tone-shock pairings, $100 \%$ reinforcement) and an unpredictable group which received independent presentations of tones and shocks. The predictable group had a mild bradycardia, which was not statistically significant when compared with the unpredictable group. Unfortunately, that study utilized one 19-h immobilization session to assess this psychological variable. Immobilization stress is such a potent stimulus that it may have obscured any more subtle influences of the psychological manipulation.

A second cardiovascular study (Miller, Grossman, Richardson, Wistow, \& Thomas, 1978) measured the myocardial uptake of technetium-99-methylene diphosphonate, a marker for tissue injury, following one 2-h session of either signaled or unsignaled shock. Uptake was significantly higher in the unsignaled group. The authors concluded that the signaled condition protected those animals from "an important psychological component of the stress"' (p. 434).

A final cardiovascular study examined the latency to both ventricular tachycardia and death during slow iv infusion of digitalis in four groups of guinea pigs (signaled shock, unsignaled shock, signal alone, and no stimuli) following seven 80-min training sessions (Natelson \& Cagin, 1981). During the digitalis infusion on the 8th day of study, the light that was associated with shock in the signaled group was illuminated on the same schedule as usual, but was not illuminated for the other groups, since it had not been presented previously to those groups. No shocks were administered on the test day. Results demonstrated that the latency to ventricular tachycardia was significantly decreased in the signaled group. There were no differences between the other groups. This study was not without problems, however. For all groups, it had been $24 \mathrm{~h}$ since the last session, and there is no reason to suspect that any of the groups (including the signaled one) would have shown a reduced latency to ventricular tachycardia in the absence of an immediately preceding session. That the signaled group showed a reduction merely reflects the associative strength previously developed between the light and shock. Since it relates to digitalis, a widely prescribed drug, this is an important observation, but it cannot serve as an adequate test of the predictability dimension.

Since rats prefer predictable to unpredictable shock, one might anticipate that predictable shock would produce less of a physiological perturbation than unpredictable shock. This prediction seems a logical extension of each of the hypotheses outlined above. In the case of the preparatory response hypothesis, the animal presumably engages in some behavioral or internal adjustment which reduces the aversiveness of the shocks. Thus, physiological functioning during the signal should be different from what it is in the absence of the signal. This would not be expected in a group of animals for which the signal had no meaning (unpredictable shock).

A similar prediction would be made for the other major hypotheses as well. In the case of the safety signal hypothesis, the absence of the signal would be expected to produce a tendency to return to normal homeostatic function in the predictable but not the unpredictable situation. In the Rescorla-Wagner model, there would be no association of the signal with fear cues in the unsignaled situation, and thus no physiological differences would be expected when a neutral signal was compared to absence of that signal. Thus, all three hypotheses would predict that physiological functioning would be more perturbed in the unsignaled or unpredictable situation.

In the current studies, groups of animals were equated on the number of shocks received per session but differed in their predictability, as signaled by a tone. During the test session, arterial blood pressure was continuously monitored in order to obtain systolic and diastolic blood pressure, as well as heart rate. In the second study, blood was obtained immediately after the session for the determination of plasma catecholamines and corticosterone. It was hypothesized, on the basis of the theoretical formulations outlined above, that the greatest departure from homeostatis would be found in the least predictable group.

\section{EXPERIMENT 1}

\section{Method}

Twenty-four male Long-Evans rats, weighing $250-300 \mathrm{~g}$, were used in this study. All animals had ad-lib access to food and water throughout the duration of the experiment. The animals were divided randomly into three groups of eight rats each. For Group $100 \%$, cutaneous electric shock (1-sec duration, .6-mA, applied to the tail) was always associated with a $30-\mathrm{sec}, 1-\mathrm{kHz}, 75-\mathrm{dB}$ tone. For Group $75 \%$, shock was associated with $75 \%$ of the tone-on and $25 \%$ of the tone-off periods. Finally, for Group 50\%, shock was equally associated with the toneon and tone-off periods.

All groups were exposed to 401 -min trials daily. Each trial consisted of a 30-sec tone-off period, followed by a 30-sec tone-on period. All shocks occurred midway through either the tone-off or tone-on period. For Groups $75 \%$ and $50 \%$, shocks were delivered randomly and according to the aforementioned percentages in the tone-off or tone-on period, with the following restrictions: (1) no trial had more than one shock, and (2) the random schedule generated in the first 20 trials was repeated in the second 20 .

Thus, all groups received 40 shocks daily, once each minute. The only difference between groups was the probability that shock would occur during the tone-on period. The animals were studied for 5 consecutive days, 40 trials per day, to stabilize the cardiovascular response to the tone-on and tone-off periods. The presentation of tones and shocks was under microprocessor control, utilizing hardware and software systems previously described (Lawler \& Buchholz, 1979; Murray \& Lawler, 1978).

On the 6th day of study, all animals were anesthetized with sodium pentobarbitol anesthesia ( $50 \mathrm{mg} / \mathrm{kg}$, ip), and a polyethylene cannula containing heparinized saline was inserted into the lower abdominal aorta, utilizing direct puncture with a 27 -ga needle. The cannula was inserted through the puncture and the tip was positioned upstream just distal to 
the inferior renal artery. The cannula was tested for patency, filled with heparinized saline, plugged with a stainless steel obturator, sutured in place, tunnelled under the skin, and exited through an incision at the nape of the neck. The animals then received procaine penicillin $(60,000$ units, im), and were given a minimum of $48 \mathrm{~h}$ recovery. To maintain patency, catheters were flushed daily with $.25 \mathrm{ml}$ of heparinized saline.

Following recovery from surgery, the animals were again exposed to 40 conditioning trials, while arterial blood pressure was monitored on-line utilizing a Narco Bio-Systems strain gauge transducer and physiograph and custom-designed microprocessor hardware and software. The strain gauges were calibrated daily using a mercury column. The physiograph output from each blood pressure channel was fed into an eight-channel, 8-bit analog-to-digital converter (Burr-Brown, Inc.) and digitized every $4 \mathrm{msec}$. The software was used to store systolic blood pressure for $5 \mathrm{sec}$. Then a new interval began, and blood pressure was stored in a new memory location. Thus, for each trial, and for each animal, there were 12 systolic blood pressure data points, each corresponding to a 5-sec mean. Data were stored in the memory of a SYM-1 microcomputer system (Synertek Systems) until the end of the session. A BASIC program was then used to format and output the data to a printer (IDS 440). All data were then hand-entered onto hard disk for statistical analysis using the BMD package on an IBM-360 computer. Preliminary analyses utilized analysis of variance for each dependent variable, and follow-up tests employed the Tukey HSD. A significance level of $p<.05$ was chosen throughout

\section{Results}

An analysis of variance for systolic blood pressure, utilizing 5-sec blocks and groups as factors, yielded main effects of both these factors $[F(11,231)=30.68, F(2,21)$ $=3.80$, respectively], as well as a significant interaction $[F(22,231)=2.61]$. Figure 1 depicts the mean systolic blood pressure for each of the groups across 5 -sec blocks. This figure suggests two conclusions: (1) The unpredictable shock group (50\%) has lower, not higher, blood pressure, and (2) the source of the interaction appears to be due to differences in responding to the 5-sec block immediately following shock (Blocks 4 and 10).

Separate analyses of variance on each block revealed significant group effects for Blocks 3-6, 8, 9, and 11, mar-

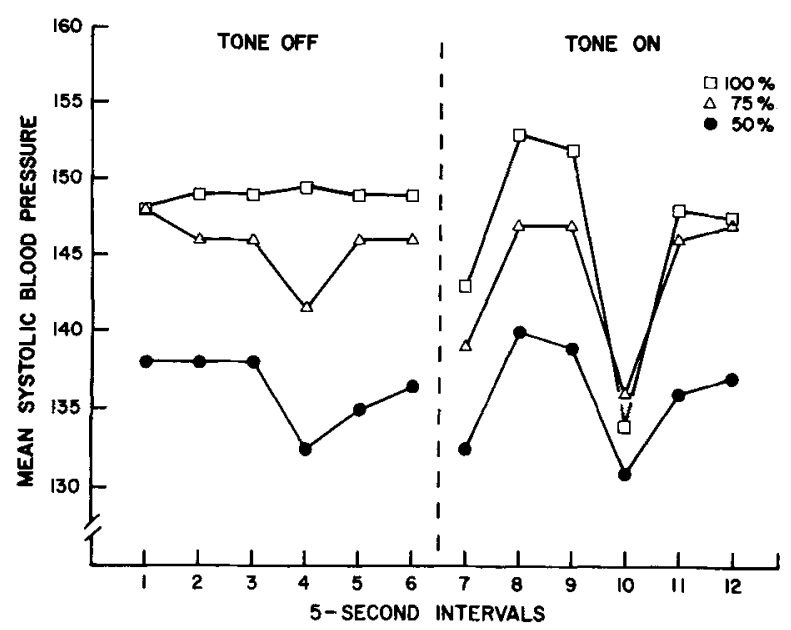

Figure 1. Mean systolic blood pressure levels during tone-off (left panel) and tone-on (right panel) periods in the $100 \%$ predictable ( $\square-$ $\square$ ), $75 \%$ predictable $(\triangle-\triangle)$, and $50 \%$ predictable groups ( $O-$ O). Each point represents the mean for a 5 -sec block collapsed across all conditioning trials. ginally significant differences $(\mathrm{p}<.10)$ for Blocks 1,2 , 7 , and 12 , and no differences at Block 10 . The source of the significant block effects was determined with Tukey HSD post hoc tests. For each of the seven blocks with significant overall $\mathrm{F}$ ratios, Tukey HSD tests revealed that the $50 \%$ group had significantly lower blood pressure than the $100 \%$ group. The $75 \%$ group in each case was intermediate and not significantly different from either of the other two groups.

\section{Discussion}

Although no previous studies have examined blood pressure changes in groups exposed to differing probabilities of cutaneous electric shock, we had predicted, on the basis of theories that seek to explain the behavioral preference for predictable shock, that the least predictable shock group $(50 \%)$ would have the highest blood pressure. In fact, their blood pressure was significantly lower at all 12 time blocks and was lower than that of the $100 \%$ group on seven of those blocks. These data do not appear to support any of the major theories of preference for predictable shock. A second study was initiated (1) to determine whether this observation was robust enough to withstand replication, (2) to expand the assessment of cardiovascular variables to include diastolic blood pressure and heart rate, and (3) to add assessments of sympathoadrenal function by determining plasma catecholamines and corticosterone.

\section{EXPERIMENT 2}

\section{Method}

Again, 24 male Long-Evans rats were used and were randomly assigned to three groups. All major aspects of the study remained the same, except for the following: (1) Software was modified to include measurement of diastolic blood pressure and heart rate; (2) since trends seemed less important than overall blood pressure levels in Experiment 1, software was further modified to determine pressure and rate for 30 sec intervals, that is, for each tone-on and tone-off period, instead of for 5-sec blocks; (3) on the day when blood pressure was recorded, the 401 -min trials consisted of 20 shock trials followed by 20 nonshock (extinction) trials; and (4) blood was obtained for plasma assays on the 5 th conditioning day before surgical intervention. The extinction trials were the first exposure of any group to trials without shock; that is, the 5-day presurgery training period was identical to that of Experiment 1 . Immediately after the end of the fifth conditioning session, approximately $5-10 \mathrm{~mm}$ of the rat's tail was rapidly clipped with a scalpel, as detailed by Bagby, McDonald, and Mass (1979), and $1 \mathrm{ml}$ blood was collected in a tube containing $1.8 \mathrm{mg}$ EGTA and $1.2 \mathrm{mg}$ glutathione. The sample was gently mixed and centrifuged in the cold for $15 \mathrm{~min}$, and plasma was stored at $-20^{\circ} \mathrm{C}$ until assayed.

Plasma catecholamines were measured in duplicate by a modification of the radioenzymatic technique described by Cryer, Santiago, and Shah (1974). Catechol-o-methyl transferase was extracted by the method described by Axelrod and Tomchick (1958). The tritium labeled S-adenosyl methionine was purchased from New England Nuclear. A 100-pg E and NE standard was run as an internal standard with each plasma sample. The coefficient of variation for the $E$ and NE internal standard averaged $5 \%$.

For corticosterone measurement, a $50 \mu \mathrm{l}$ aliquot of plasma was extracted twice using $2 \mathrm{ml}$ of methylene chloride. The extracted sample was then resuspended in $1.5 \mathrm{ml}$ of phosphate buffered saline (PBS). Cor- 
ticosterone content was determined by a modified RIA procedure developed by Mayes, Furuyama, Kim, and Nugent (1970). Corticosterone antiserum (anticorticosterone-3 BSA serum, No. 377) was obtained from Gordon D. Niswender of the Department of Physiology and Biophysics, Colorado State University. The $\left(1,2{ }^{3} \mathrm{H}(\mathrm{N})\right)$-corticosterone was procured from New England Nuclear (Boston, MA). The intra-assay coefficient of variation was less than $5 \%$.

All statistical analyses utilized the BMD package on the IBM- 360 computer. The mixed-design analysis utilized one between-subjects factor (groups) and two within-subjects factors (tone and blocks). The group factor had three levels $(100 \%, 75 \%, 50 \%)$ and the tone factor had two levels (off and on). The blocks factor had eight levels, each consisting of a five-trial block. Thus, for the 40 trials, the first four blocks consisted of tone and shock trials, while the last four blocks consisted of tone-only extinction trials. All post hoc tests utilized the Tukey HSD. The level of significance chosen was again $\mathrm{p}<.05$.

\section{Results}

Systolic blood pressure. Analyses revealed significant effects of tone $[\mathrm{F}(1,23)=10.95]$ and blocks $[\mathrm{F}(7,161)$ $=47.83]$, but not groups $[\mathrm{F}(2,23)=2.54, \mathrm{p}=.10]$. There were significant interactions of blocks $\times$ groups $[F(14,161)=1.65]$ and tone $\times$ blocks $[F(7,161)=5.34]$, as well as a triple interaction of groups $\times$ tones $\times$ blocks $[F(14,161)=2.33]$. In a follow-up analysis, a blocks $\times$ groups interaction was found for the tone-off period $[F(14,161)=2.04]$ but not the tone-on period $[F(14,161)$ $=1.32, \mathrm{p}=.20 \mathrm{]}$. That interaction is depicted in Figure 2 .

As can be seen, the $100 \%$ group had a higher systolic blood pressure than the other two groups for all trial blocks, including extinction. This was confirmed statistically using the Tukey HSD. Group $100 \%$ was significantly higher than either of the other two groups for all trial blocks, including extinction. Groups $75 \%$ and $50 \%$ did not differ from each other for any trial block.

As another means of depicting the triple interaction, separate ANOVAs were performed for each group. Only Group $100 \%$ showed a main effect of tone $[F(1,8)=$ 6.06]. Although all groups showed a main effect of blocks, there was a difference between groups in the tone $x$ blocks interaction. This interaction was significant for

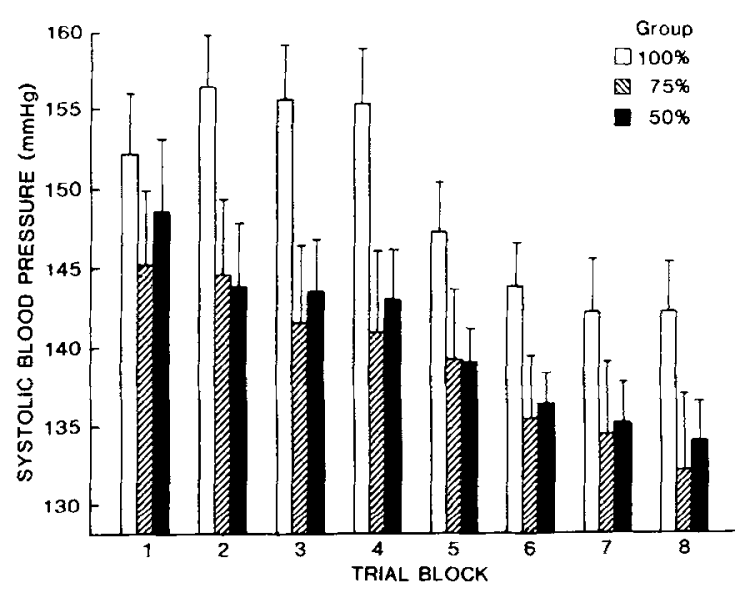

Figure 2. Mean ( \pm SEM) systolic blood pressure levels during acquisition (Trial Blocks 1-4) and extinction (Trial Blocks 5-8) in the $100 \%$ predictable $(\square), 75 \%$ predictable $(\$)$, and $50 \%$ predictable (D) groups. Each trial block represents the mean blood pressure levels for five conditioning trials in each of the eight animals.
Groups $100 \%$ and $75 \%[\mathrm{~F}(7,56)=5.85$ and $\mathrm{F}(7,56)=$ 2.98 , respectively], but not for Group $50 \%(\mathrm{~F}<1.0)$.

In summary, the systolic blood pressure data revealed a triple interaction of groups $\times$ tones $\times$ blocks. An interaction of tones $\times$ blocks was found for the tone-off but not the tone-on period. Tukey HSD tests revealed that Group $100 \%$ had significantly higher pressures than the other two groups at all blocks, including extinction. These differences ranged from 6.9 to $14.9 \mathrm{mmHg}$. Although Group $75 \%$ had lower pressures than Group 50\% in six of the eight trial blocks, these differences were not statistically significant. Groups $100 \%$ and $75 \%$ showed a tone $\times$ blocks interaction, but Group $50 \%$ did not. The source of the tone $\times$ blocks interaction for the former groups was a tendency for tone-off minus tone-on difference scores to be higher during extinction than during acquisition, an effect not observed in Group 50\%.

Diastolic blood pressure. Analyses revealed significant effects of tone $[F(1,23)=10.50]$ and blocks $[F(7,161)=22.08]$, but not groups $(F<1.0)$. There were significant interactions of blocks $\times$ groups $[F(14,161)=1.84]$ and tones $\times$ blocks $[F(7,161)=$ 1.95], but no triple interaction $[F(14,161)=1.38, p=$ .17]. The pattern of responding was similar to that for systolic pressure, but less pronounced. Consequently, no figure is presented.

Post hoc analyses utilizing Tukey HSD tests revealed that Group $100 \%$ had significantly higher pressure than either of the other two groups on all trial blocks except the first. The range of these differences varied between 2.1 and $8.0 \mathrm{mmHg}$. Group $75 \%$ and Group $50 \%$ differed only on the first block of extinction.

Heart rate. Analyses revealed significant effects of groups $[F(2,23)=5.69]$, tones $[F(1,23)=23.96]$, and blocks $[F(7,161)=6.32]$ and groups $\times$ tones $\times$ blocks $[F(14,161)=4.62]$. The source of the triple interaction was sought by separate ANOVAs for the tone-on and toneoff periods, which were not informative regarding the source of interaction, since both failed to show significant blocks $\times$ groups interactions.

A set of three additional ANOVAs, one for each group, revealed that there were significant block and tone $X$ block interactions for each analysis. However, one difference was found: Although Groups $100 \%$ and $75 \%$ had significant main effects of tone $[F(1,8)=15.53$ and $F(1,8)$ $=30.60$, respectively], Group $50 \%$ did not $(F<1.0)$.

Overall, the $75 \%$ group had the highest heart rate (428 bpm), followed by the 50\% (392 bpm) and 100\% groups (391 bpm). Tukey HSD post hoc tests revealed that the $75 \%$ group had higher heart rates than either of the other two groups for all trial blocks, including extinction. Groups $100 \%$ and $50 \%$ showed two significant, but opposite, differences in Trial Blocks 3 and 4. In general, heart rates in the $75 \%$ group tended to be approximately $35 \mathrm{bpm}$ higher than those in the other two groups.

Sympathoadrenal response. Table 1 presents mean $( \pm$ standard error) NE, E, and corticosterone values for each of the three groups. One-way analysis of variance revealed no significant group effects for $\mathrm{NE}$ or $\mathrm{E}[\mathrm{F}(2,26)=.25$ 
Table 1

Mean ( \pm Standard Error) Norepinephrine (NE), Epinephrine (E), and Corticosterone (CORT) Responses in Three Groups Differing in the Probability of Cutaneous Electric Shock During a Signal

\begin{tabular}{rccc}
\hline Group & NE $(\mathrm{pg} / \mathrm{ml})$ & $\mathrm{E}(\mathrm{pg} / \mathrm{ml})$ & CORT $(\mu \mathrm{g} / \mathrm{dl})$ \\
\hline $100 \%$ & $558.0 \pm 51.6$ & $424.2 \pm 42.1$ & $42.4 \pm 6.2$ \\
$75 \%$ & $577.2 \pm 67.8$ & $402.5 \pm 58.8$ & $51.5 \pm 2.2$ \\
$50 \%$ & $513.6 \pm 73.4$ & $346.3 \pm 50.5$ & $34.4 \pm 5.5$ \\
\hline
\end{tabular}

and $\mathrm{F}(2,26)=.61$, respectively], and a marginal effect for corticosterone $[F(2,21)=2.65, p<.10]$. A Tukey HSD post hoc follow-up for the corticosterone data revealed that the source of this marginal effect was itself marginal: there was a trend $(p<.10)$ for the $50 \%$ and $75 \%$ groups to differ.

\section{DISCUSSION}

The fact that rats will choose to receive predictable over unpredictable shock led to the hypothesis that, in the present studies, animals receiving totally predictable shock would show smaller blood pressure, heart rate, catecholamine, and corticosterone responses than rats receiving unpredictable shock. This hypothesis, although consistent with all the major theories that attempt to explain the behavioral preference for signaled shock, was not supported by either experiment. Animals receiving predictable electrical shock had significantly higher systolic and diastolic blood pressure than animals receiving unpredictable shock.

Both heart rate and corticosterone values showed an inverted-U relation to predictability, with the intermediate group $(75 \%)$ having the highest value. An explanation for the discrepancy between the blood pressure and heart rate data may rest with the observation that the heart rate may have been low in the $100 \%$ and $50 \%$ groups for different reasons. In the 50\% group, low heart rates may have accompanied low pressure due to the fact that this paradigm was less aversive and the rats were less aroused. In the case of the $100 \%$ group, the elevated blood pressure may have elicited the baroreceptor reflex, thus holding down heart rate, which otherwise would have been much higher.

In the only other study of heart rate and predictability (Caul et al., 1972), no differences were found in the preCS heart rate between the predictable and unpredictable groups (Groups III and V of the Caul et al., 1972, study). This baroreceptor hypothesis is further supported by the mild bradycardia noted by Caul et al. (1972) in response to the CS.

The failure of the present study, and of the other cardiovascular studies mentioned, to support theories that attempt to explain the preference for predictable shock leads to consideration of whether other dependent physiological variables are more supportive. Bassett et al. (1973) found elevated corticosterone levels in rats receiving predictable shock when the shock occurred at either regular or irregular intervals. Paré (1964) observed elevated adrenal weight to body weight ratios and elevated adrenal ascorbic acid concentrations (a marker for ACTH release) in animals receiving predictable shock. Davis and Levine (1982) found that corticosterone values were higher in animals receiving signaled shock early in the session but that groups were equal by the end of the 30-min session (Experiment 4).

On the other hand, Simpson, Wilson, DiCara, Jarrett, and Carroll (1975) failed to find any differences between predictable and unpredictable groups, as did Hennessy, King, McClure, and Levine (1977) and Davis and Levine (1982). The only study to demonstrate a greater elevation in corticosterone values in the unpredictable group is that of Weiss (1970). Although Weiss (1970) suggested that some differences between studies might be explained on the basis of the inadvertent availability of escape from grid shock, by jumping or postural changes, subsequent studies, including the present one, utilized tailshock. The present study found an inverted-U relationship between corticosterone and predictability. However, there were no differences in corticosterone levels between the least predictable and most predictable groups.

The most consistent results relating physiological indices to predictability have been obtained with studies utilizing ulceration as the dependent variable. Sawrey (1961), Seligman (1968), Seligman and Meyer (1970), and Weiss (1970) all found that unpredictable shock leads to greater stomach ulceration than predictable shock. The only discrepant studies have been those of Gliner (1972), who showed no differences in his signal-yoked and yoked groups (predictable and unpredictable, respectively), and Caul et al. (1972). In the latter study, there was a trend for the unpredictable group to have higher ulcer scores, but it was not statistically significant. The use of immobilization stress in the Caul et al. (1972) study may have obscured differences between groups, since the procedure itself is a potent ulcerogenic stimulus. Rats in other studies cited above were subjected to less restrictive restraint.

It thus appears that, except for studies of ulceration, there is little physiological evidence to support theories about behavioral preference for signaled shock. In part, this reflects the complexities of physiological response systems. No one physiological variable can be used as an index of the "stress" response. It also reflects the need either to revise existing theories about preference for signaled shock or to develop separate theories that seek to explain the body of physiological data.

\section{REFERENCES}

Aввотт, B., \& BADIA, P. (1979). Choice for signaled over unsignaled shock as a function of signal length. Joumal of the Experimental Analysis of Behavior, 32, 409-417.

AXELROD, J., \& TOMCHICK, R. (1958). Enzymatic o-methylation of epinephrine and other catechols. Journal of Biological Chemistry, 233, 702-705

Badia, P., Culberrson, S., \& Lewis, P. (1971). The relative aversiveness of signaled vs unsignaled avoidance. Joumal of the Experimental Analysis of Behavior, 16, 113-121.

BADIA, P., HARSH, J., \& ABBOTT, B. (1979). Choosing between predict- 
able and unpredictable shock conditions: Data and theory. Psychological Bulletin, 86, 1107-1131.

Bagby, S. P., McDonald, W. J., Mass, R. D. (1979). Serial reninangiotensin studies in spontaneously hypertensive and Wistar-Kyoto rats: Transition from normal-to-high-renin status during the established phase of spontaneous hypertension. Hypertension, 1, 347-354.

Bassett, J. R., Cairncross, K. D., \& KING, M. G. (1973). Parameters of novelty, shock predictability and response contingency in corticosterone release in the rat. Physiology \& Behavior, 10, 901-907.

Biederman, G. B., Furedy, J. J., \& BeatTy, J. G. (1981). The preference-for-signaled-shock phenomenon: Classical conditioning paradigms. Psychological Record, 31, 357-369.

Caul, W. F., Buchanan, D. C., \& Hays, R. C. (1972). Effects of unpredictability of shock on incidence of gastric lesions and heart rate in immobilized rats. Physiology \& Behavior, 8, 669-672.

Cryer, P. E., Santiago, J. W., \& Shah, S. (1974). Measurement of norepinephrine and epinephrine in small volumes of human plasma by a single isotopic derivative method: Response to the upright posture. Journal of Clinical Endocrinology and Metabolism, 39, 1025-1029.

DAvis, H., \& Levine, S. (1982). Predictability, control, and the pituitaryadrenal response in rats. Journal of Comparative and Physiological Psychology, 96, 393-404.

FANSELOW, M. S. (1979). Naloxone attenuates rat's preference for signaled shock. Physiological Psychology, 7, 70-74.

Furedy, J. J., \& Biederman, G. B. (1976). Preference for signaled shock phenomenon: Direct and indirect evidence for modifiability factors in the shuttlebox. Animal Learning \& Behavior, 4, 1-5.

Gliner, J. A. (1972). Predictable vs unpredictable shock: Preference behavior and stomach ulceration. Physiology \& Behavior, 9, 693-698.

Hennessy, J. W., King, M. G., MCCluRe, T. A., \& Levine, S. (1977). Uncertainty, as defined by the contingency between environmental events, and the adrenocortical response of the rat to electric shock. Joumal of Comparative and Physiological Psychology, 91, 1447-1460.

Lawler, J. E., Barker, G. F., Hubbard, J. W., \& Schaub, R. G. (1981). Effects of stress on blood pressure and cardiac pathology in rats with borderline hypertension. Hypertension, 3, 496-505.

LAWLER, J. E., \& BuchHOLz, R. A. (1979). A software program for the control of aversive conditioning schedules using the KIM-1 microprocessor. Behavior Research Methods \& Instrumentation, 11, 218-220.

LOCKARD, J. A. (1963). Choice of warning signal or no warning signal in an unavoidable shock situation. Journal of Comparative and Physiological Psychology, 56, 526-530.

Mayes, D., Furuyama, S., Kim, D. C., \& Nugent, C. A. (1970). A radioimmunoassay of plasma aldosterone. Journal of Clinical Endocrinology and Metabolism, 30, 682-685.
Miller, D. G., Grossman, Z. D., Richardson, R. L., Wistow, B. W., \& Thomas, F. D. (1978). Effect of signaled versus unsignaled stress on rat myocardium. Psychosomatic Medicine, 40, 432-434.

MuRRAY, D. M., \& LAWLER, J. E. (1978). A KIM-1 microprocessor interface for aversive conditioning applications using multiple subjects. Behavior Research Methods \& Instrumentation, 10, 334-339.

NAtelson, B. H., CAGIN, N. A. (1981). The role of shock predictability during aversive conditioning in producing psychosomatic digitalis toxicity. Psychosomatic Medicine, 43, 191-197.

PARÉ, W. P. (1964). The effect of chronic environmental stress on stomach ulceration, adrenal function, and consummatory behavior in the rat. Journal of Psychology, 57, 143-151.

Perkins, C. C. (1968). An analysis of the concept of reinforcement. Psychological Review, 75, 155-172.

Rescorla, R. A., \& Wagner, A. R. (1972). A theory of Pavlovian conditioning: Variations in the effectiveness of reinforcement and nonreinforcement. In A. H. Black \& W. F. Prokasy (Eds.), Classical conditioning II: Current research and theory (pp. 64-99). New York: Appleton-Century-Crofts.

SAWREY, W. (1961). Conditioned responses of fear in the relationship to ulceration. Journal of Comparative and Physiological Psychology, 54, 347-348.

Seligman, M. E. P. (1968). Chronic fear produced by unpredictable electric shock. Journal of Comparative and Physiological Psychology, 66, 402-411.

Seligman, M. E. P., Maier, S. F., \& Solomon, R. L. (1971). Unpredictable and uncontrollable aversive events. In F. R. Brush (Ed.), Aversive conditioning and learning (pp. 347-400). New York: Academic Press.

Seligman, M. E. P., \& Meyer, B. (1970). Chronic fear and ulcers in rats as a function of the unpredictability of safety. Journal of Comparative and Physiological Psychology, 73, 202-207.

Simpson, C. W., Wilson, L. G. M., DiCara, L. V., Jarrett, K. J., \& CARroll, B. J. (1975). Stress-induced ulceration in adrenalectomized and normal rats. Bulletin of the Psychonomic Society, 6 , 189-191.

WEISs, J. M. (1970). Somatic effects of predictable and unpredictable shock. Psychosomatic Medicine, 32, 397-408.

WeIss, J. M. (1977). Ulcers. In J. D. Maser \& M. E. P. Seligman (Eds.), Psychopathology: Experimental models (pp. 232-269). San Francisco: Freeman.

(Manuscript received April 11, 1983; revision accepted for publication June 18,1984 .) 